\title{
Benigne Prostatahyperplasie
}

\section{Pflanzenduo bremst auch die Entzündung}

_ Chronische Entzündungsprozesse gelten heutzutage als möglicher Trigger für Entstehung und Progression von benigner Prostatahyperplasie (BPH) und benignem Prostatasyndrom (BPS). Sie könnten ein mögliches Ziel für antiphlogistische Therapieansätze sein. Neue Daten zeigen, dass die Phyto-Kombination aus Sägepalme(Sabal)- und Brennnessel(Ur-

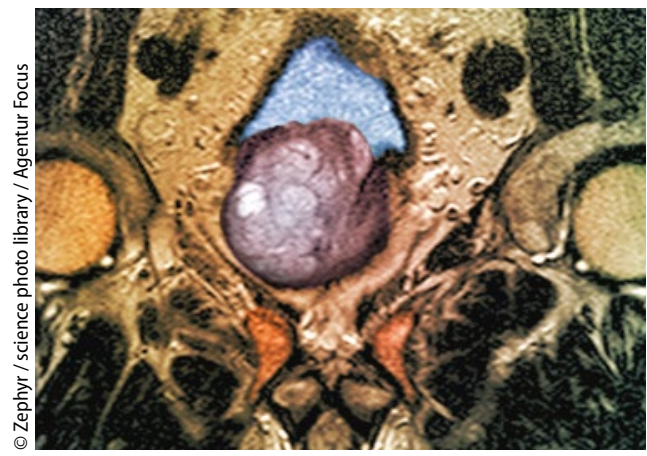

Wie lange geht das noch gut?
tica)-Extrakten PRO 160/120 die Genexpression wichtiger Entzündungsmediatoren und Wachstumsfaktoren in einem BPH-Modell hemmt.

\section{Reduktion der Zytokin-Expression}

In der von Dr. Stefano Palea, ein Pharmakologe aus Toulouse, durchgeführten Studie wurde ein BPH-Modell, das der Pathologie beim Menschen nahekommt, verwendet. Die BPH wurde bei Ratten mittels Sulpirid-Verabreichung induziert. Die Genexpression wichtiger Entzündungsmediatoren wie Interleukin-2, Interleukin-15, Cyclooxygenase-2, induzierbarer NO-Synthase sowie von Early Growth Response-Protein-1 und Epidermal Growth Factor waren im Prostatagewebe unbehandelter Kontrolltiere deutlich erhöht.

Unter Therapie mit PRO 160/120 (Prostagutt ${ }^{\circledast}$ forte) war die Genexpres- sion beider Interleukine sowie der Entzündungsenzyme und Wachstumsfaktoren signifikant geringer als in der Kontrollgruppe $(\mathrm{p}<0,05)$. Finasterid unterdrückte nur die Expression eines dieser sechs Gene (EGF). Die Ergebnisse legen nahe, dass PRO 160/120 bei $\mathrm{BPH}$ sowohl eine anti-inflammatorische als auch eine anti-proliferative Wirkung hat, fasste Palea die Ergebnisse zusammen.

Bei Patienten besserte die PhytoKombination BPS-Beschwerden wie Nykturie vergleichbar wie gängige synthetische BPS-Präparate. Die Wirksamkeit von PRO 160/120 wird in den deutschen und europäischen Leitlinien zur BPS-Therapie dokumentiert.

Dr. Martina Utzt

- Expertenworkshop "Anti-inflammatory approaches to BPS", Kongress der Deutschen Gesellschaft für Urologie; Leipzig, September 2016 (Veranstalter: Schwabe)

\section{Postprandiale Blutzuckerkontrolle}

\section{Besser und kostengünstiger mit Insulin aspart}

_ Der postprandiale Anstieg der Blutzuckerwerte ist nachweislich ein Risikofaktor für kardiovaskuläre Ereignisse. Für diese Beobachtung besteht Dr. Andreas Liebl, Bad Heilbrunn, zufolge ein hoher Grad an Evidenz. „Dass es nicht gleichgültig ist, mit welchem Insulin diese Kontrolle vorgenommen wird, konnte eine retrospektive Datenbankanalyse mit mehr als 6.000 Patienten belegen“, so der Diabetologe [Rathmann W, Kostev K. Diabetes Obes Metab. 2013;15:358-63].

\section{Weniger makrovaskuläre Erkrankungen}

Ausgewertet wurden Daten von Patienten aus allgemeinmedizinischen Praxen in Deutschland. Zu den Einschlusskriterien zählten eine Diabetesdauer von $\geq 2$ Jahren vor einem mikro- oder mak- rovaskulären Ereignis, eine Mindesttherapiedauer von $\geq 30$ Monaten, die kontinuierliche Behandlung in der gleichen Praxis und ein Patientenalter $>40$ Jahre.

Das Ergebnis lautete, dass das Risiko für makrovaskuläre Ereignisse unter Insulin aspart (NovoRapid ${ }^{\circledR}$ ) um 15\% niedriger war als unter der Therapie mit kurz wirksamen Humaninsulinen ( $\mathrm{p}=0,01)$. Das Risiko für einen Schlaganfall oder eine transitorische ischämische Attacke war um $42 \%$ geringer, das Myokardinfarktrisiko um $31 \%$ niedriger und das Risiko für eine periphere arterielle Verschlusskrankheit (PAVK) um 20\% niedriger.

\section{Gesundheitsökonomische Analyse}

Auf Basis der Patientendaten wurde ergänzend eine gesundheitsökonomische
Analyse vorgenommen [2]. Das reduzierte Risiko für makrovaskuläre Erkrankungen und die damit einhergehende geringere Anzahl an Begleiterkrankungen bei einer Therapie mit Insulin aspart gegenüber Humaninsulin wurde dabei ins Verhältnis mit den jeweils entstehenden Kosten der beiden Therapieformen gesetzt.

„Daraus errechnete sich ein Gewinn an qualitätsadjustierten Lebensjahren bei gleichzeitiger Kostenersparnis im deutschen Gesundheitswesen durch den Einsatz von Insulin aspart im Vergleich zu Humaninsulin“, fasste Liebl die Ergebnisse zusammen.

Dr. Silke Wedekind

- Springer Medizin Expertenworkshop "Postprandialer Blutzucker - wird seine Bedeutung unterschätzt?"; München, September 2016 (Veranstalter: Novo Nordisk) 